\title{
Interactive comment on "Spin-up Characteristics with Three Types of Initial Fields and the Restart Effects on the Forecast Accuracy in GRAPES Global Forecast System” by Zhanshan Ma et al.
}

\section{Anonymous Referee \#2}

Received and published: 12 October 2020

The manuscript investigates the influence of spin-up and restart in a global weather forecast system GRAPES. Such a topic is important, as careful handling of those technical issues can greatly improve the accuracy of weather prediction. By comparing different spin-up and restart methods, the authors gain important knowledge of the forecasting system, such as that the GRAPES with its own analysis field performs better than the one using NCAR final reanalysis (FNL) data for the cold start in the spin-up. The paper contains useful information for model development and usage. I recommend its publication with GMD, pending on some minor comments below.

About the experiment setup. To better illustrate the differences between three experi- 
ments, which are of great importance to this paper, can the authors use a schematic plot to show how the three runs were performed and what are the key input data. Also, it should be explicitly stated in the Section 1.2, why those three experiments were conducted, or in other words, what we expect to learn by comparing them. The three-hour lag confuses me a little bit.

It is unclear to me what is the current protocol for spin-up and restart strategies used by CMA that uses GRAPES_GFS to conduct the daily weather prediction as well as the extreme weather prediction like typhoons. According to this study, is there any modification needed on the protocol?

Is the total grid number of cloud (TGNC) related with the total cloud fraction? The latter is a more common term. Also, $1.0 \mathrm{e}-4 \mathrm{~g} \mathrm{~kg}-1$ threshold of cloud sounds an arbitrary choice. Are the results sensitive to this threshold definition?

Fig. 11a, why g00 and g21 are identical before 42 Hour and then become different abruptly?

Interactive comment on Geosci. Model Dev. Discuss., https://doi.org/10.5194/gmd-2020-177, 2020. 